\title{
Anomalous dispersion of transparent atomic two- and three-level ensembles
}

\author{
A. Rocco, ${ }^{1}$ A. Wicht, ${ }^{1, *}$ R.-H. Rinkleff, ${ }^{2}$ and K. Danzmann ${ }^{1,2}$ \\ ${ }^{1}$ Max-Planck-Institut für Gravitationsphysik, Albert-Einstein-Institut, Teilinstitut Hannover, Callinstrasse 38, D-30167 Hannover, Germany \\ ${ }^{2}$ Institut für Atom- und Molekülphysik, Abteilung Spektroskopie, Universität Hannover, Callinstrasse 38, D-30167 Hannover, Germany
}

(Received 20 June 2002; published 11 November 2002)

\begin{abstract}
The absorption and dispersion properties of a driven two-level atomic system probed by a weak field have been measured simultaneously with a phase modulated Mach-Zehnder interferometer. We find that the resonant absorption and the negative dispersion are reduced with respect to the nondriving case by a factor of 490 and 47 , respectively, which is in good agreement with the theory. This means a $\sim 10$-fold enhancement of the amount of dispersion provided per amount of residual absorption. The experimental values are compared to the requirements that have to be fulfilled for the realization of a broadband, high finesse optical cavity. Finally, we propose a specific degenerate three-level system that overcomes some of the shortcomings of the driven two-level atom.
\end{abstract}

DOI: 10.1103/PhysRevA.66.053804

PACS number(s): 42.62.Fi, 32.80.Qk

\section{INTRODUCTION}

The steady-state absorption of a weak and resonant probe beam can be strongly modified by the means of additional optical fields. The absorption can be reduced or even eliminated or can rather be increased by the presence of a coupling beam exciting a linked transition. The reduction of the atomic absorption named electromagnetically induced transparency (EIT) results from the coherent interaction of the medium with a resonant driving field $[1,2]$. The opposite effect, the enhancement of the absorption resulting from atomic coherence induced by optical radiation, is designated electromagnetically induced absorption [3]. The modification of the absorptive properties of an atomic vapor generally also leads to a modification of the dispersive properties.

In the last decade, the study of the dispersive properties of coherently prepared media was always under attention because of fundamental and practical interest. An extremely slow group velocity of light [4-6] or even "stopping" of light $[7,8]$ were demonstrated using alkaline atoms. In these experiments the absorption was strongly suppressed and the dispersion was steep and normal due to the coherent population trapping (CPT) between the two ground-state hyperfine levels. As a complementary application superluminal group velocities were realized based on strong negative (anomalous) dispersive media [9]. Further, anomalous dispersive transparent media have been considered for the realization of broadband, high finesse optical cavities [10], for which the anomalous dispersion is supposed to cancel the variation of optical wavelengths with frequency inside the cavity (white light cavity).

Recently, we have given a comparative theoretical analysis of five different atomic schemes with respect to their performance as negative dispersive transparent media [11]. These schemes were the strongly driven two-level atom (TLA) and the degenerate three-level atom (DTLA) [12], two closely spaced gain lines and two double- $\Lambda$-schemes with incoherent pumping. There it was shown, that a strongly

*Electronic address: wicht@pallas.amp.uni-hannover.de driven TLA or a DTLA can be used to realize negative dispersive transparent media. The DTLA shows various features: in a closed transition, depending on the angular momentum, negative dispersion and electromagnetically induced absorption (EIA) [13], negative dispersion without absorption, or normal dispersion of a CPT resonance [14] can be found. As opposed to a closed transition, EIA cannot be established in an open transition.

The aim of this paper is first to investigate experimentally the requirements for the realization of a white light cavity based on the strongly driven TLA. Second, we focus at an alternative scheme in order to overcome shortcomings inherent to the strongly driven TLA.

For our experiment the Ca resonance line at $422.6 \mathrm{~nm}$ was chosen to investigate the absorption and the index of refraction spectra of a weak probe field interacting with a driven TLA. The loss and the phase shift were measured simultaneously with a phase modulated Mach-Zehnder interferometer $[15,16]$. The experimental results verified the theoretical analysis of the strongly driven TLA well. For a detailed theoretical analysis of a resonant driving TLA, the reader is referred to Ref. [17] and references therein. We were able to reach a regime of driving field intensities at which the suppression of absorption overcomes the suppression of dispersion. Thus, we demonstrated experimentally that negative dispersive transparent media are feasible, which are a requirement for the implementation of white light cavities. The experimental results also pointed out that for the realization of a white light cavity driving field intensities would be required, which were not accessible at this wavelength. One of the goals of using an alternative system, i.e., the DTLA, is to overcome this problem. Therefore, the DTLA is compared to the driven TLA within the context of realizing negative dispersive transparent media.

\section{EXPERIMENT}

The experimental observation of the absorption and the index of refraction spectra of a strongly driven TLA were 


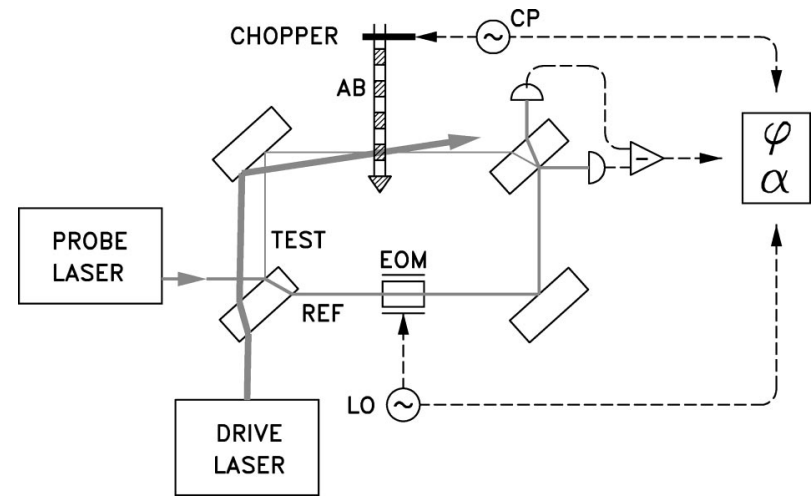

FIG. 1. Experimental setup of the phase modulated MachZehnder interferometer. EOM is the electro-optic modulator, LO is the local oscillator for the EOM $(1.8 \mathrm{MHz}), \mathrm{CP}$ is the local oscillator of the atomic beam chopper $(1.7 \mathrm{kHz})$, and $\mathrm{AB}$ is the atomic beam.

performed on the $4 s^{2}{ }^{1} S_{0}-4 s 4 p{ }^{1} P_{1}$ transition of Ca atoms in an atomic beam. For the investigation, a spectroscopic method was developed which consists of a Mach-Zehnder interferometer with a phase modulated reference arm [16]. This way it was possible to perform a highly sensitive simultaneous measurement of both the phase shift and the absorption profiles. The interferometer was mechanically and acoustically decoupled from the environment by an intravacuum two-stage isolation mount and by operating it completely in an UHV chamber. The vacuum chamber was continuously pumped to less than $10^{-7}$ Torr. To help maintain the vacuum and to reduce the calcium vapor background, a liquid-nitrogen-cooled trap was connected to it. The experimental setup was similar to the one previously presented [16], therefore only a brief account will be given here stressing details pertinent to the present experiment.

The pump and probe fields were generated from the output of two identical grating laser systems, each based on an injection-locked master-slave system. The master oscillators were laser diodes with an optical feedback from a holographic grating in Littrow configuration. The output from each of the slave lasers was frequency doubled in a singleresonant external cavity using $\mathrm{KNbO}_{3}$ crystals to provide the desired wavelength. About $20 \mathrm{~mW}$ of blue power at $423 \mathrm{~nm}$ was obtained using the fundamental power of $120 \mathrm{~mW}$. For a more detailed information on the laser system the reader is referred to Ref. [18]. The frequency of the pump laser was locked to the Ca transition $4 s^{2}{ }^{1} S_{0}-4 s 4 p{ }^{1} P_{1}$ by means of frequency modulation spectroscopy setup performed on a $\mathrm{Ca}$ heat pipe. A fraction of the output of the corresponding master laser was frequency shifted by an acousto-optic modulator to a fixed frequency position relative to the atomic transition $(-180 \mathrm{MHz})$. It was used to phase lock the probe laser frequency at a tunable offset frequency $(-100 \mathrm{MHz}$ $<\Delta f<2 \mathrm{GHz})$.

The pump and probe beams were focused at the atomic beam inside the Mach-Zehnder interferometer, see Fig. 1. The intensities were controlled with neutral density filters. At the interaction zone the probe beam had a power of 0.022 $\mathrm{mW}$ and a beam radius of about $0.134 \mathrm{~mm}$. The maximum driving field power available in the interaction zone was about $10 \mathrm{~mW}$ and the beam radius about $0.338 \mathrm{~mm}$ corresponding to a saturation parameter of $S \approx 20$. The pump and probe fields both were linearly and identically polarized and were propagating almost into the same direction, slightly diverging by less than $20 \mathrm{mrad}$ to allow separate detection and preventing the pump beam from entering the Mach-Zehnder interferometer. The two beams were incident at right angles with respect to the atomic beam. In the interaction zone the atomic beam had a diameter of about $2 \mathrm{~mm}$ at a collimation of 1:200. At a typical oven temperature of $T=1200 \mathrm{~K}$, the residual transversal Doppler width of the atoms was reduced to a value below the natural line width.

To measure absorption and phase shift spectra a small fraction of the probe beam power was split off at the input beam splitter of the Mach-Zehnder interferometer. It was directed into the test arm of the interferometer to interact with the strongly driven atoms of the atomic beam, thus undergoing a definite phase shift and absorption. The main probe power was directed into the reference arm of the interferometer, where the optical field was phase modulated $\left(f_{\mathrm{LO}}\right.$ $=1.8432 \mathrm{MHz}$ with a phase modulation index of $M \approx 1)$. For locking the interferometer on a dark fringe, the error signal of the balanced output of the interferometer was used after demodulation at $f_{\mathrm{LO}}$. The feedback signal of the corresponding servoloop was applied to the phase modulator in the reference arm. It contains information about the phase shift imprinted onto the test field during the interaction with the atoms of the mechanically chopped $\left(f_{\mathrm{CP}}=1.7 \mathrm{kHz}\right)$ atomic beam. The detection of the servosignal with a dualphase lock-in amplifier at $f_{\mathrm{CP}}$ revealed the phase shift of the optical probe field. The absorption signal was directly deduced from the low-frequency component of the balanced output of the interferometer by a second dual-phase lock-in amplifier demodulating at $f_{\mathrm{CP}}$. With regard to the phase measurement, the interferometer was operated as an active null instrument and therefore cross talking was avoided between the absorption and the index of refraction signal even for large phase shifts and for large absorption.

To investigate a true two-level scheme a linearly polarized pump laser was used to drive the $\Delta m=0$ transition of the $\mathrm{Ca}$ resonance line and the identically polarized probe laser was scanned across the same transition. In order to maximize the suppression of the absorption and to realize a negative dispersive transparent medium, the field intensity of the pump laser was varied and the absorption and phase shift was recorded for different driving field Rabi frequencies $\Omega_{\mathrm{D}}$ $=d E_{0} /(2 \hbar)$, where $d$ is the electric dipole moment and $E$ $=E_{0} \cos \left(\omega_{\mathrm{D}} t\right)$ is the electric component of the driving optical field. From each of these measurements, absorption and dispersion at the atomic resonance were determined.

\section{RESULTS AND DISCUSSION}

The experimental results are shown in Fig. 2. The dispersion of the index of refraction $n^{\prime}=\partial_{\omega} n$ and the power absorption coefficient $\alpha$ of the probe beam at atomic resonance are given vs the driving field Rabi frequency. The dispersion is given in units of $1 / \gamma$, where $\gamma=2 \pi(34 \mathrm{MHz}) 0.7$ is the exited-state population decay rate. The factor 0.7 has been 


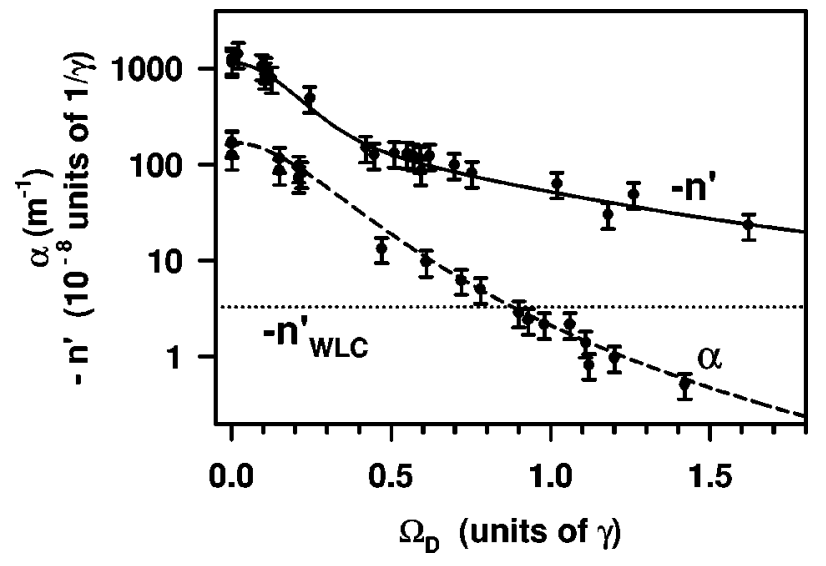

FIG. 2. Experimental results for absorption and negative dispersion at atomic resonance vs driving field Rabi frequency for the $\mathrm{Ca}$ resonance transition at $423 \mathrm{~nm}$. Negative dispersion given in units of $10^{-8} / \gamma$, where $\gamma=0.7 \times 2 \pi 34 \mathrm{MHz}$. $n_{\mathrm{WLC}}^{\prime}$ denotes the dispersion required for the realization of a white light cavity for this specific scheme. The solid and dashed lines correspond to the theoretical model for an atomic density of $N_{0}=2 \times 10^{9} \mathrm{~cm}^{-3}$.

included to account for the fact that the effective line width determined in our experiment was smaller than the value given in the literature by 30\% [19]. This discrepancy has been attributed to radiation trapping effects [15]. All data points have been normalized to an atomic density of $N_{0}=2$ $\times 10^{9} \mathrm{~cm}^{-3}$, which is typical for our data. The solid and dashed lines correspond to the theoretical model [11]. Also shown is the value $n_{\mathrm{WLC}}^{\prime}=-1 / \omega_{0}=3.3 \times 10^{-8} / \gamma$, which is required for the realization of a white light cavity at an optical wavelength of $\lambda=423 \mathrm{~nm}$ [15].

To compare different atomic schemes with respect to their capability of providing negative dispersion without absorption, it is worthwhile to discuss the amount of dispersion available per amount of residual absorption. We call the ratio of the normalized dispersion $n^{\prime} / n_{\mathrm{WLC}}^{\prime}=\partial_{\delta} n /\left(-1 / \omega_{0}\right)$ to the power absorption coefficient $\alpha$ figure of merit: $\Theta_{\mathrm{FOM}}$ $=\left(n^{\prime} / n_{\mathrm{WLC}}^{\prime}\right) / \alpha$. Here, $\omega_{0}$ is the cavity resonance frequency that we assume to be identical to the atomic resonance frequency. Please note, that resonant dispersion and absorption scale like $n^{\prime} \propto 1 / \Omega_{\mathrm{D}}^{2}$ and $\alpha \propto 1 / \Omega_{\mathrm{D}}^{4}$ for the strongly driven TLA ( [15], see also Fig. 4). As expected, the largest figure of merit $\Theta_{\text {FOM }}$ has been achieved at the largest driving field Rabi frequency available $\left(\Omega_{\mathrm{D}}=1.6 \gamma\right)$, which corresponds to a saturation parameter of $S \approx 20$. At this driving level the figure of merit is enhanced by a factor of 10.5 with respect to the case without driving $\Omega_{\mathrm{D}}=0$. Further, even for the strongest driving feasible the dispersion was still larger than the dispersion $n_{\mathrm{WLC}}^{\prime}$ by a factor of 7. Hence, in principle, one could improve the figure of merit by another factor of 7 and still maintain the desired dispersion if more powerful lasers were available.

However, a realistic scenario for the experimental implementation of a white light cavity would require a figure of merit of $\theta_{\mathrm{FOM}} \approx 1000 \mathrm{~m}$ [15], which is $\approx 50$ times larger than the largest figure of merit achieved so far. This could be achieved with driving field powers beyond $1 \mathrm{~W}$ at $\lambda$
$=423 \mathrm{~nm}$, which is a serious constrain in experimental terms. Further, there is another potential drawback inherent to the strongly driven TLA: in the strong driving regime, the atoms scatter photons at rate $\gamma / 2$ causing dissipation of both the driving and the probing field. Therefore, the strongly driven TLA would not be a good choice if one had to care about quantum noise properties of the probing or driving field, e.g., for quantum-nondemolition type of experiments relying on the strong coupling between both the fields mediated by the atoms.

Next we propose an alternative approach to overcome both of the problems mentioned above. It is based on a DTLA which has been discussed within the context of enhancing the index of refraction of transparent media [20]. This scheme could be realized by means of a $J=1 \rightarrow J^{\prime}=1$ transition, where the circularly polarized components of a linearly polarized driving field coherently couple the ground states $J=1, M=-1$ and $M=+1$ via the exited state $J^{\prime}$ $=1, M^{\prime}=0$. If no magnetic field is present, the magnetic sublevels are degenerate and a single driving field is twophoton resonant thus establish strong ground-state coherence and CPT. The CPT will be governed by residual ground-state decoherence at rate $\gamma_{0} \ll \gamma$. Please note, that due to the specific choice of angular momenta $\left(J=J^{\prime}=1\right)$ and opticalfield polarizations $\left(\sigma^{+}+\sigma^{-}\right)$, all atoms will be pumped out off the $|M=0\rangle$ Zeeman sublevel of the ground state and will effectively be transferred into the corresponding $M= \pm 1$ Zeeman sublevels.

The corresponding spectra for a weak probe field of identical polarization point out a remarkable similarity to the spectra of the strongly driven TLA. An example is given in Fig. 3, which shows the power absorption coefficient and the index of refraction vs the probe field detuning for the strongly driven TLA (graph $A$ ) and the DTLA (graph $B$ ) under equivalent conditions. The data for the DTLA have been multiplied by $\gamma / \gamma_{0}=10^{3}$ to ease the comparison. According to Fig. 3(b), the dispersion at atomic resonance for the DTLA is reduced by a factor of $10^{3}$ with respect to the strongly driven TLA, but the reduction of resonant absorption is much more strongly pronounced [Fig. 3(a), inset]. Hence the DTLA provides a larger figure of merit than the strongly driven TLA under equivalent conditions. Further, as CPT is established in the DTLA, the exited-state population is strongly reduced. Hence, dissipation and loss of both the driving and the probing field are avoided in the limit of $\gamma$ $\gg \gamma_{0} \rightarrow 0$.

Figure 4 provides a comparison of both schemes with respect to their performance as negative dispersive transparent media: resonant absorption $\alpha_{0}$ and dispersion $n_{0}^{\prime}$ of both schemes are shown vs the driving field Rabi frequency $\Omega_{D}$. We find three basic results:

(i) For both schemes $\alpha_{0} \propto 1 / \Omega_{\mathrm{D}}^{4}$ and $n_{0}^{\prime} \propto 1 / \Omega_{\mathrm{D}}^{2}$ for $\Omega_{\mathrm{D}}$ $\gg \gamma$, so that the figure of merit scales like $\Omega_{\mathrm{D}}^{2}$ and negative dispersion without absorption can be realized.

(ii) The amount of negative dispersion provided by the DTLA at a specific driving field Rabi frequency is smaller than the dispersion provided by the strongly driven TLA by a factor of $\gamma /\left(\sqrt{2} \gamma_{0}\right)$. However, if one adjusts the driving field Rabi frequencies independently in order to achieve the same 

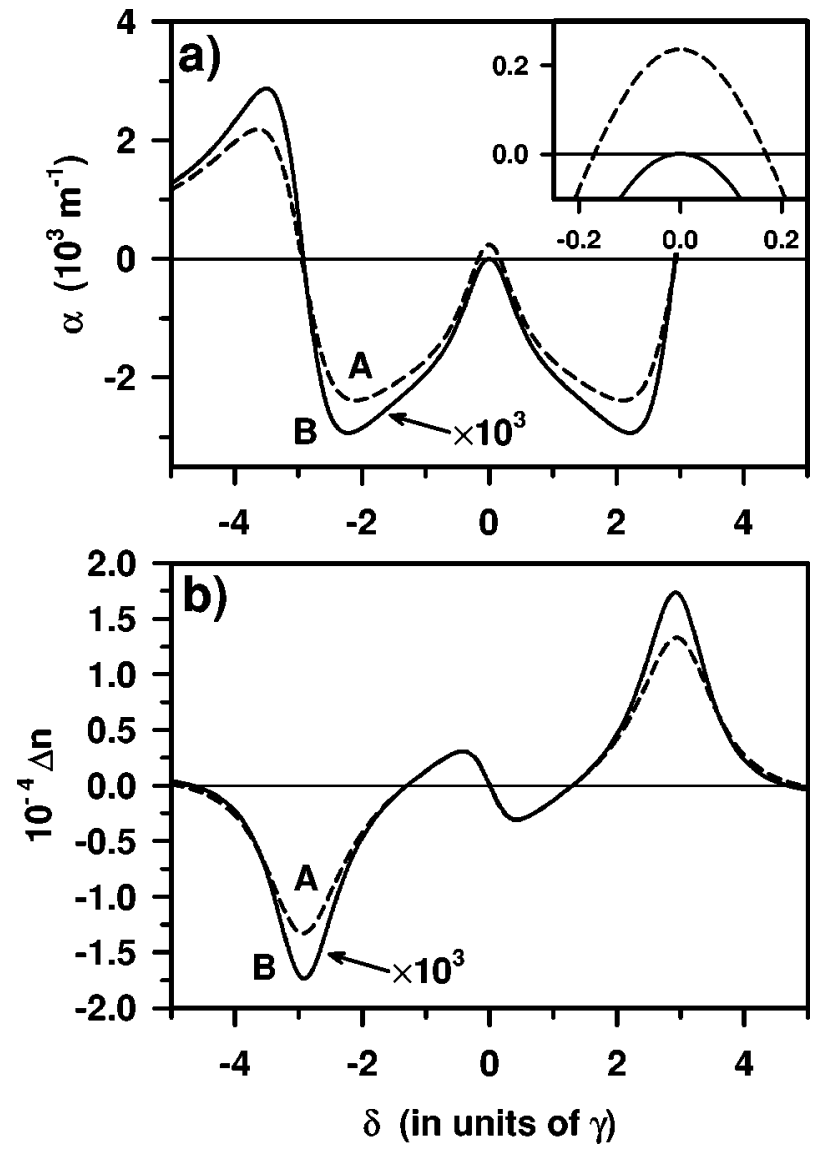

FIG. 3. Power absorption coefficient (a) and index of refraction (b) vs probe field detuning for the strongly driven TLA (graph $A$ ) and the DTLA (graph $B$ multiplied by $10^{3}$ ). The inset of (a) shows the resonance in detail. Equivalent parameters are used for both systems: atomic density $N_{0}=10^{12} \mathrm{~cm}^{-3}$, transition wavelength $\lambda$ $=423 \mathrm{~nm}$. For the strongly driven TLA $\Omega_{\mathrm{D}}=1.5 \gamma$. For DTLA, $\Omega_{\mathrm{D}}=\sqrt{2} 1.5 \gamma$ and ground-state decoherence rate $\gamma_{0}=10^{-3} \gamma$. Graph $A$ corresponds to the $\mathrm{Ca}$ resonance transition used in the experiment.

figure of merit for both schemes, the corresponding amount of dispersion provided by the DTLA is smaller than the amount provided by the strongly driven TLA only by a factor of 4 .

(iii) Therefore, the DTLA basically shows the same performance as the strongly driven TLA, but provides a certain figure of merit at a driving field Rabi frequency significantly smaller than for the strongly driven TLA. This has been indicated by the arrows in Fig. 4. Finally, it should be noted that the DTLA shows a regime of modest driving where positive dispersion is provided.

\section{CONCLUSION}

We have shown that it is possible to realize negative dispersive transparent media that provide enough anomalous dispersion to compensate for the variation of wavelength with frequency in vacuum. This is a requirement for the

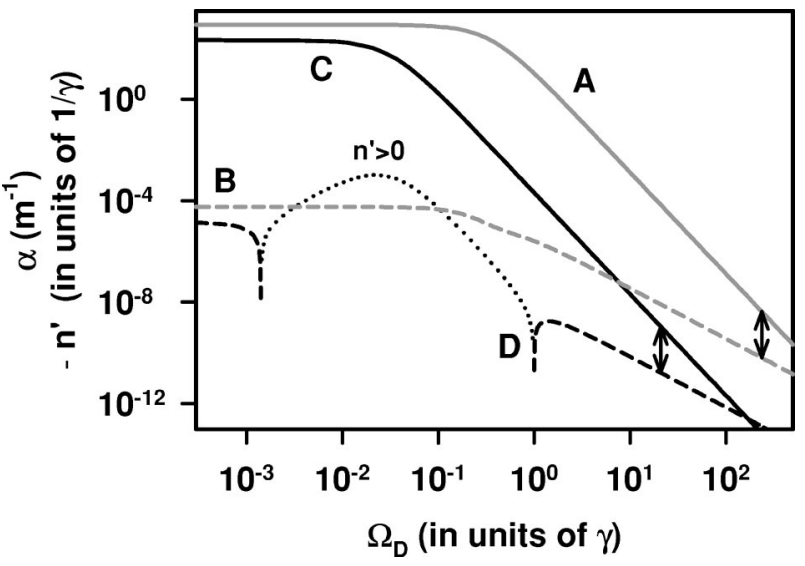

FIG. 4. Performance of the TLA and the DTLA for realization of negative dispersion without absorption. Shown are the resonant absorption and the resonant negative dispersion of the strongly driven TLA (graphs $A$ and $B$, respectively) and of the DTLA (graphs $C$ and $D$, respectively). Parameters are $N_{0}=10^{10} \mathrm{~cm}^{-3}, \lambda=423 \mathrm{~nm}$ for both schemes, and $\gamma_{0}=10^{-3} \gamma$ for the DTLA. The arrows indicate points corresponding to identical figures of merit.

implementation of broadband, high finesse optical cavities (white light cavities). The maximum figure of merit that has been achieved is $\theta_{\mathrm{FOM}}=21 \mathrm{~m}$ at a typical dispersion of 15 $\times 10^{-10} / \mathrm{MHz}$ and absorption of $0.33 \% / \mathrm{cm}$. For comparison, the amount of dispersion required for the implementation of a white light cavity is $2.2 \times 10^{-10} / \mathrm{MHz}$. The figure of merit of a sample of simple $\mathrm{Ca}$ atoms (i.e., without driving) is $\theta_{0}, \mathrm{FOM}=2.0 \mathrm{~m}$. Hence, we have been able to provide 10.5 times more dispersion per amount of residual absorption than the most simple system would provide. Further, we have proposed an alternative atomic scheme, i.e., a DTLA which should provide larger figures of merit than the strongly driven TLA at a given driving field intensity while maintaining basically the same amount of dispersion at a given figure of merit. In addition to relaxing the requirements for the driving field intensities, with the DTLA one would also avoid dissipation of both the driving and the probing field. This system could be realized, for example, with the $1 s 2 s{ }^{3} S_{1} \rightarrow 1 s 2 p{ }^{3} P_{1}$ transition or with the $1 s 2 s$ ${ }^{3} S_{1} \rightarrow 1 s 3 p{ }^{3} P_{1}$ transition of $\mathrm{He}$ at $\lambda=1083 \mathrm{~nm}$ and $\lambda$ $=388.9 \mathrm{~nm}$, respectively. Please note, that although the $1 s 2 s{ }^{3} S_{1}$ state is not the ground state, it would nevertheless provide long-living Zeeman coherences that are required for the realization of strong negative dispersion. This is due to the fact that the $1 s 2 s{ }^{3} S_{1}$ state is metastable. Therefore circularly polarized components of the linearly polarized driving field would efficiently couple the $M=-1$ and $M=+1$ sublevels of the $2 s$ state via the exited state $M^{\prime}=0$. Provided that there is no magnetic field, a probe field of identical polarization would show the desired spectra.

\section{ACKNOWLEDGMENT}

This work was supported by the SFB 407 of the Deutsche Forschungsgemeinschaft. 
[1] S.E. Harris, Phys. Today 50(7), 36 (1997).

[2] E. Arimondo, in Progress in Optics $X X X V$, edited by E. Wolf (Elsevier Science, Amsterdam, 1996), p. 257.

[3] A.M. Akulshin, S. Barreiro, and A. Lezama, Phys. Rev. A 57, 2996 (1998).

[4] L.V. Hau, S.E. Harris, Z. Dutton, and C.H. Behroozi, Nature (London) 397, 594 (1999).

[5] D. Budker, D.F. Kimball, S.M. Rochester, and V.V. Yashchuk, Phys. Rev. Lett. 83, 1767 (1999).

[6] M.M. Kash, V.A. Sautenkov, A.S. Zibrov, L. Hollberg, G.R. Welch, M.D. Lukin, Yu. Rostovtsev, E.S. Fry, and M.O. Scully, Phys. Rev. Lett. 82, 5229 (1999).

[7] Ch. Liu, Z. Dutton, C.H. Behroozi, and L.V. Hau, Nature (London) 409, 490 (2001).

[8] D.F. Phillips, A. Fleischhauer, A. Mair, R.L. Walsworth, and M.D. Lukin, Phys. Rev. Lett. 86, 783 (2001).

[9] A. Dogariu, A. Kuzmich, and L.J. Wang, Phys. Rev. A 63, 053806 (2001); L.J. Wang, A. Kuzmich, and A. Dogariu, Nature (London) 406, 277 (2000).

[10] A. Wicht, K. Danzmann, M. Fleischhauer, M. Scully, G. Müller, and R.-H. Rinkleff, Opt. Commun. 134, 431 (1996).

[11] A. Wicht, R.-H. Rinkleff, L. Spani Molella, and K. Danzmann, Phys. Rev. A (to be published).
[12] This scheme was called "degenerate two-level system" in Ref. [11] in order to follow the terminology used by Lezama et al. in Ref. [13].

[13] A. Lezama, S. Barreiro, A. Lipsich, and A.M. Akulshin, Phys. Rev. A 61, 013801 (1999); A. Lipsich, S. Barreiro, A.M. Akulshin, and A. Lezama, ibid. 61, 053803 (2000).

[14] A.M. Akulshin, S. Barreiro, and A. Lezama, Phys. Rev. Lett. 83, 4277 (1999).

[15] A. Wicht, M. Müller, R.-H. Rinkleff, A. Rocco, and K. Danzmann, in Ode to a Quantum Physicist, edited by W. E. Lamb, W. Schleich, and H. Walther (North-Holland, Amsterdam, 2000), p. 107.

[16] A. Wicht, M. Müller, V. Quetschke, R.-H. Rinkleff, A. Rocco, and K. Danzmann, Appl. Phys. B: Lasers Opt. B70, 821 (2000).

[17] C. Szymanowski, A. Wicht, and K. Danzmann, J. Mod. Opt. 44, 1373 (1997).

[18] O.S. Brozek, V. Quetschke, A. Wicht, and K. Danzmann, Opt. Commun. 146, 141 (1998).

[19] G. Zinner, T. Binnewies, F. Riehle, and E. Tiemann, Phys. Rev. Lett. 85, 2292 (2000).

[20] H. Friedmann and A.D. Wilson-Gordon, Opt. Commun. 98, 303 (1993). 